\title{
New vistas in organoelement chemistry
}

\author{
Scott E. Denmark*, Beritte L. Christenson, Stephen P. O'Connor and Noriaki \\ Murase \\ Department of Chemistry, University of Illinois at Urbana-Champaign, Urbana, \\ Illinois, 61801, USA
}

\begin{abstract}
A selective and efficient protocol for the catalytic asymmetric cyclopropanation of allylic alcohols has been developed. The reaction involves preformation of the ethylzinc alkoxide of the alcohol and reaction with the Furukawa/Simmons-Smith reagent. Zinc iodide has been shown to have a beneficial effect on the rate and enantioselectivity of the reaction. The structural variability in the allylic alcohol as been examined and the reaction was found to proceed smoothly with both $E$ and $Z$ - allylic alcohols with one or two $\beta$-substituents, but $\alpha$-substitution was not well tolerated.
\end{abstract}

The asymmetric cyclopropanation of alkenes has become an area of considerable interest in the past decade. While considerable advances in the catalytic cyclopropanation of simple alkenes using diazoacetate as the carbenoid source are on record, 1 a general method for catalytic asymmetric methylene transfer is still lacking. Early studies on cyclopropanation of asymmetrically modified substrates such as ketals, ${ }^{2}$ acetals, ${ }^{3}$ enol ethers ${ }^{4}$ and allylic alcohols ${ }^{5 a}$ were rewarding. . More recently Charette has described a stoichiometrically modified reagent which is capable of high levels of enantioselectivity. $5 \mathrm{~b}$

Our interest in developing a catalytic enantioselective cyclopropanation of allylic alcohols which employed the Furukawa modification of the Simmons-Smith reaction led us to investigate the solution and solid state structure of bis(iodomethyl)zinc. ${ }^{6}$ We recently described the optimization of a protocol for the enantioselective cyclopropanation of cinnamyl alcohol (1) with this reagent. using the bis(methanesulfonamide) of $(R, R)-1,2$-cyclohexanediamine (2) as a catalyst (Scheme 1). We first established a set of control conditions which led to a low conversion in the absence of catalyst. In the presence of $10 \mathrm{~mol} \%$ of the diethylzinc complex of 2, the reaction proceeded rapidly under the same conditions but with disappointing enantiomeric excess. This was especially surprising since Kobayashi

\section{Scheme 1}

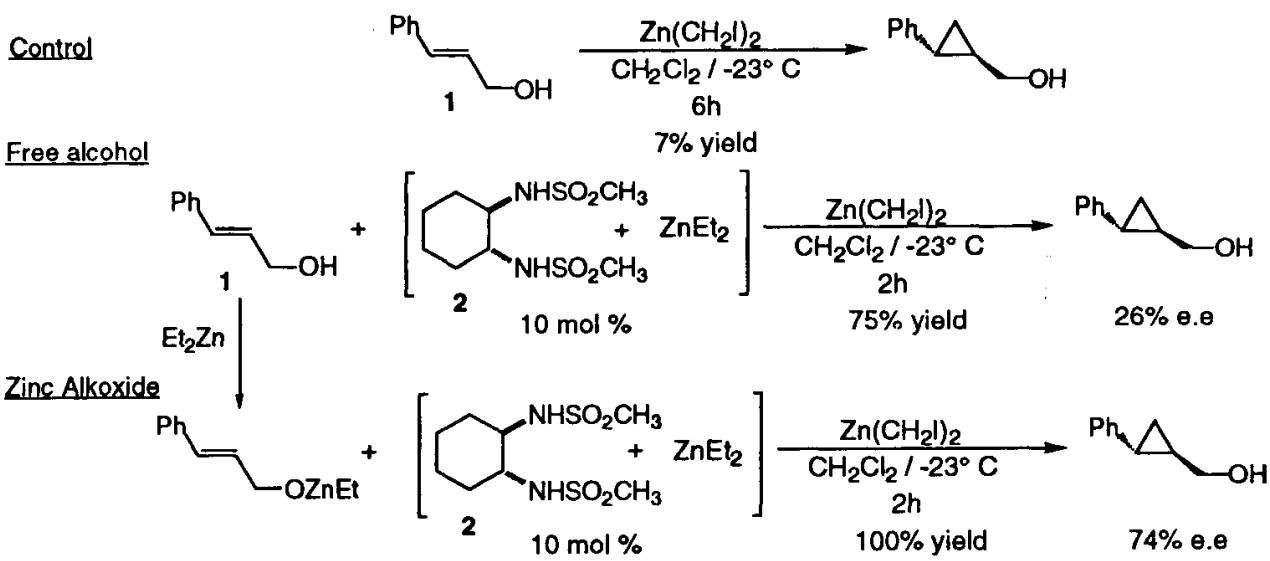


has previously reported a similar cyclopropanation of 1 with the bis(benzenesulfonamide) derivative of $(R, R)$-1,2-cyclohexanediamine in $78 \%$ enantiomeric excess. ${ }^{7}$ After careful scrutiny it was demonstrated the pre-formation of the ethylzinc alkoxide is crucial for achieving high enantioselectivity. ${ }^{8}$ In addition, control and spectroscopic experiments have revealed that the zinc salt of the sulfonamide is the active form of the chiral promoter, although its structure is still uncertain. When the $N, N$-dimethyl derivative of 2 was employed (no active NH's) the product was formed at the same rate as in an unpromoted reaction and was racemic thus providing further support that the zinc sulfonamide is the active species.

An extensive optimization of the sulfonamide residue as well as the basic structure of the diamine component did not produce a catalyst more selective than the simple bis(methanesulfonamide) (2). $9 \mathrm{~b}$ However, as part of the optimization, it was found that the reaction displayed an induction period characteristic of autocatalysis (, , Figure 1 ). Since $\mathrm{ZnI}_{2}$ is the only necessary by-product in this reaction it was reasonable to speculate the this salt was involved in the formation of a catalytically active species as the reaction proceeds. Indeed, when zinc iodide was added at the beginning of the experiment, the induction period was eliminated and the enantioselectivity increased (with $100 \mathrm{~mol} \%$ of $\mathrm{ZnI}_{2}, \mathbf{a}$ ).

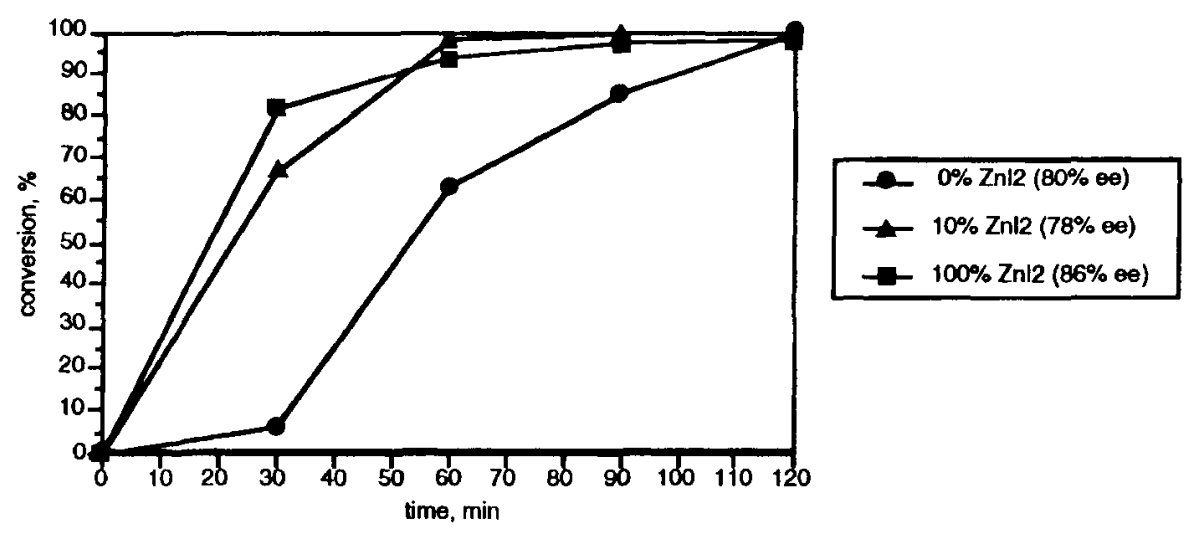

Figure 1. Effect of added zinc iodide on the rate and enantioselectivity of cyclopropanation of 1 .

In addition, we have demonstrated that zinc iodide is also necessary for the formation of the catalytically active species which is stereochemically determinant. This is crucial since externally added zinc iodide also has been shown to accelerate the unpromoted reaction. Thus, as clearly shown in Figure 2 , without additional $\mathrm{ZnI}_{2}$, not only is there an induction period in rate $(\Delta)$, but more importantly we have demonstrated that the enantiomeric excess of the product changes with time (๑). When $\mathrm{ZnI}_{2}$ is added at the outset, both the induction period and the time dependence of enantiomeric excess are eliminated.

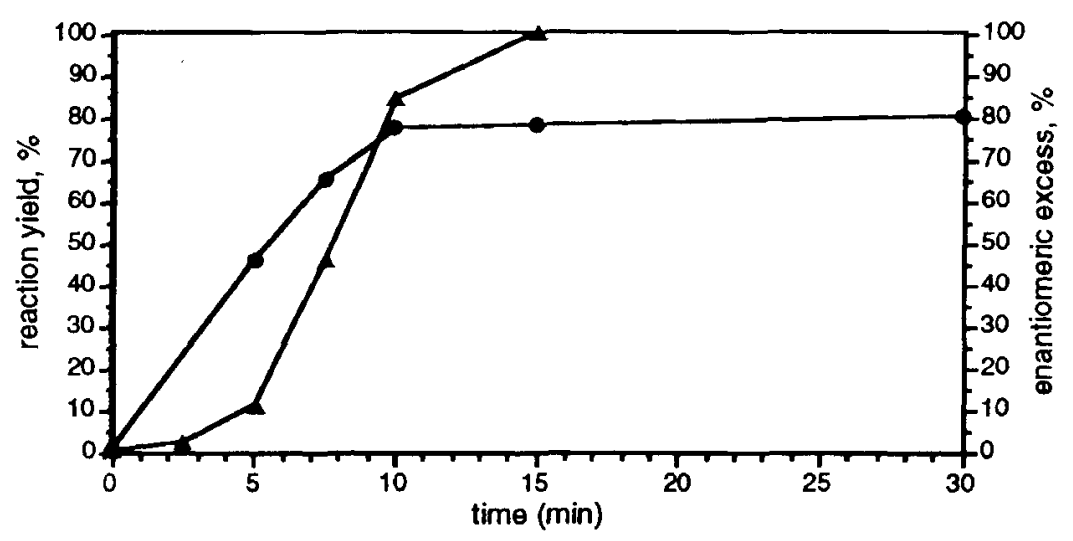

Figure 2. Time dependence of yield $(\Delta)$ and enantiomeric excess $(\bullet)$ of 3 without added $\mathrm{ZnI}_{2}$. 
At this stage, it was clear that at least four forms of zinc are needed for the reaction: the zinc alkoxide of 1 , the zinc salt of 2 , bis(iodomethyl) zinc, and zinc iodide. To gain further insight into the combination of these species to form the reactive complex, we carried out two additional mechanistic experiments. First, the methyl ether of cinnamyl alcohol was employed as the substrate. The rate of the

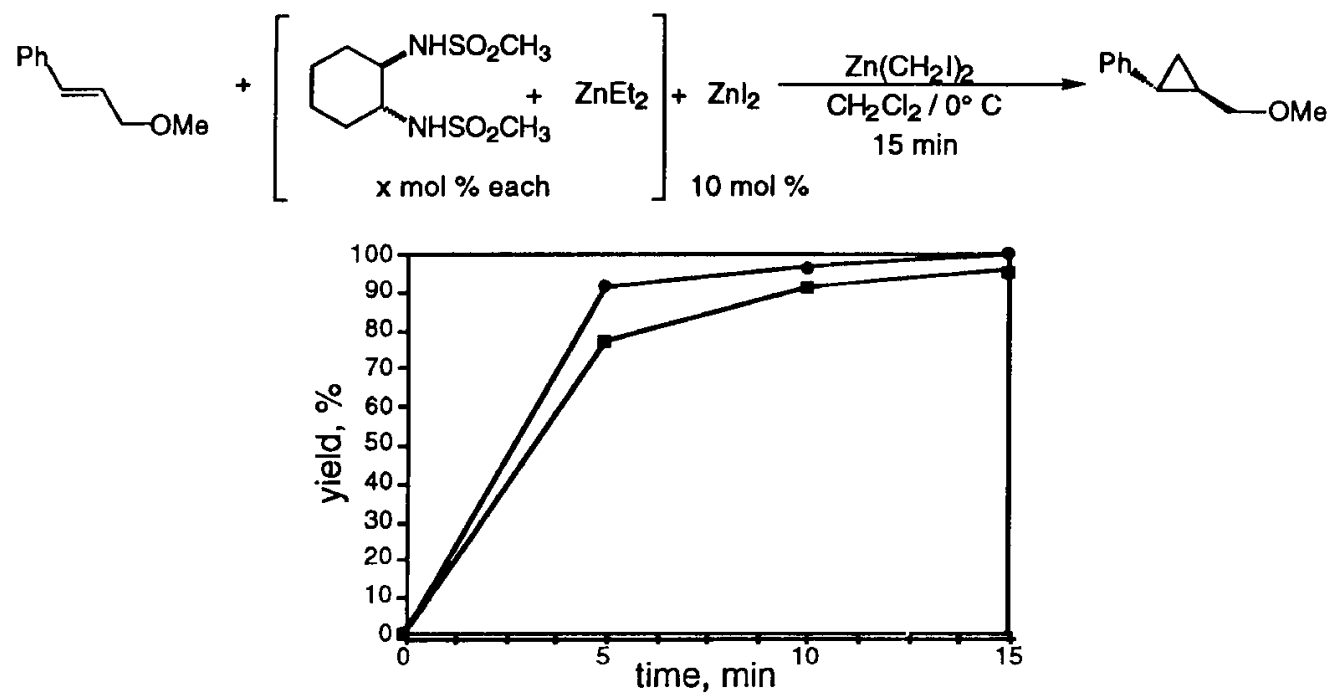

Figure 3. Cyclopropanation of cinnamyl methyl ether with $(\bullet)$ and without $(\boldsymbol{\square})$ promoter 2.

reaction was independent of the presence of the promoter and, not surprisingly, the product was racemic. This suggested that the zinc sulfonamide complex interacts with the zinc alkoxide in the substrate 1 , not the reagent. If the reagent were asymmetrically modified, a difference in rate and some level of enantioselectivity would have been expected in the methyl ether as well.

In a second experiment, we investigated the ethylidenation of cinnamyl alcohol by the use of bis(1-iodoethyl)zinc ${ }^{9}$ under the standard reaction conditions (Scheme 2). A control experiment in the absence of promoter gave the ethylidenation products in a 71/29, distal/proximal ratio. In the presence of promoter, this ratio changed slightly. Analysis of the enantiomeric composition of both diastereomers was possible by the use of chiral gas chromatography. It was found that the minor (proximal) isomer was more enantiomerically enriched $(61 \%$ ee) in the $(1 R, 2 R, 3 R)$ isomer. The major (distal) diastereomer was much less enantiomerically enriched $(12 \% \mathrm{ee})$ but interestingly the major enantiomer was found to be the $(1 R, 2 R, 3 S)$ isomer. Thus, the same enantioface of cinnamyl alcohol reacted preferentially, again supporting the notion that the substrate is modified by the promoter, not the reagent. In the latter case, the same configuration at $\mathrm{C}(3)$ would have been expected in the major enantiomer in the two diastereomers. 10

Control

Scheme 2

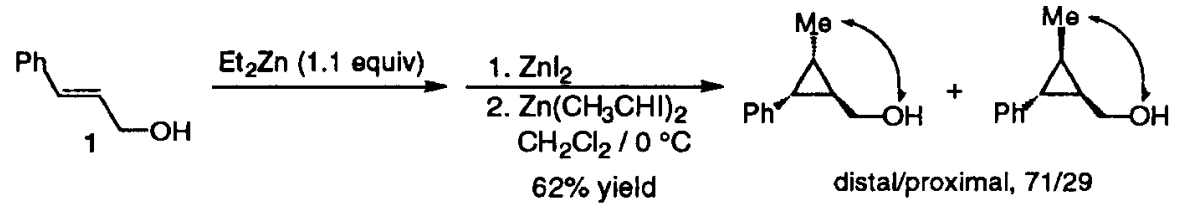

Promoted

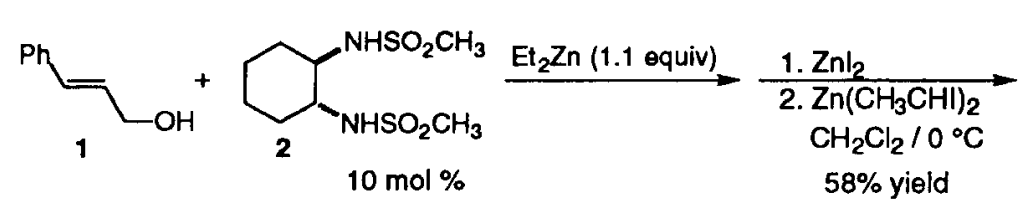

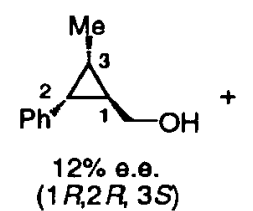

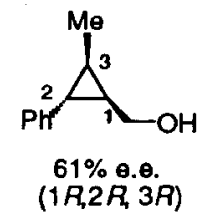

distal/proximal, $65 / 35$ 


\section{Scheme 3}

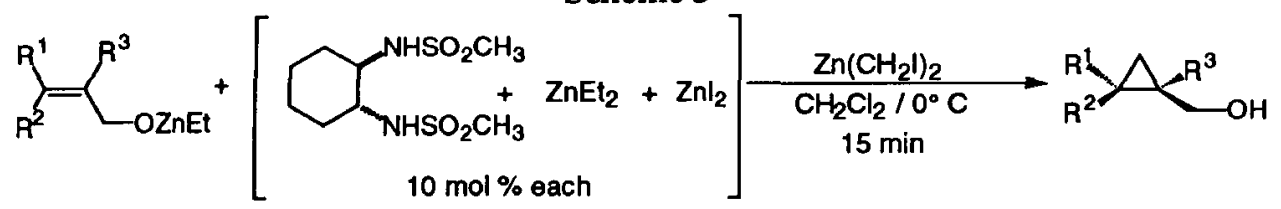

While a reasonable mechanistic picture is currently difficult to formulate due to the absence of structural information on the nature of the reactive intermediates, the preparative scope and utility of the cyclopropanation has nonetheless been explored. Application of the standard reaction protocol shown in Scheme 3 to a series of allylic alcohols has revealed interesting trends (Charts 1 and 2).

\section{Chart 1}

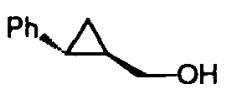

$91 \%(80 \% \theta \theta)$

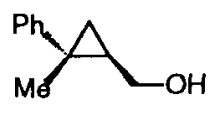

$91 \%(73 \%$ өө)

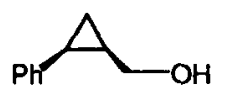

$81 \%(81 \% \theta \theta)$

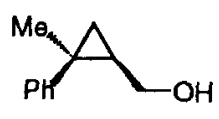

$88 \%(81 \%$ eө)

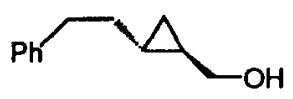

$89 \%(81 \% \theta \theta)$

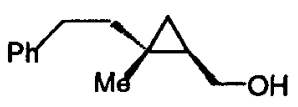

$94 \%(79 \%$ ee)

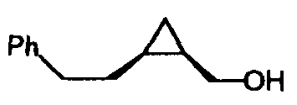

$93 \%(72 \% \theta \theta)$

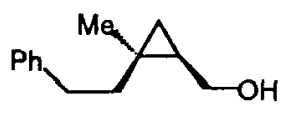

$98 \%(66 \% \ominus \theta)$

The results of catalytic asymmetric cyclopropanation of $\beta$-mono and $\beta, \beta$-disubstituted allylic alcohols are collected in Chart 1 . The yields of analytically pure products along with the enantiomeric excesses of the products are shown. The reactions all proceeded smoothly and in high yield for all substrates in this subclass. The alkene can be both $E$ and $Z$ configured and can be conjugated or substituted with saturated residues. Multiple substitution at the $\beta$-terminus is well tolerated (66-81\% ee).

The catalytic asymmetric cyclopropanation of $\alpha, \beta$-disubstituted and trisubstituted allylic alcohols was less successful (Chart 2). While the yields were excellent, the enantioselectivities were poor. The problem with this subclass is most likely the steric congestion around the zinc alkoxide and the resultant $\mathrm{A}^{1,2}$ strain that might interfere with the appropriate coordination of the chiral promoter or its preferred alignment. In addition, one example of a homoallylic alcohol is shown which also gave a poorly enantioselective reaction presumably due to the distance of the controller group from the alkene.

Chart 2

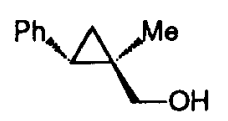

$90 \%(5 \% \theta \theta)$

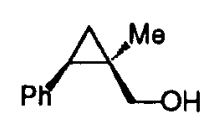

$97 \%(10 \% \theta \theta)$

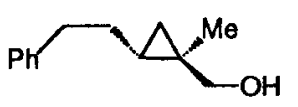

$98 \%(26 \%$ ee)

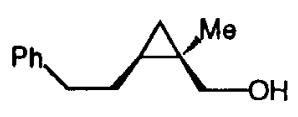

$90 \%(50 \% \theta \theta)$

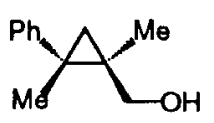

$85 \%(43 \% \theta \theta)$

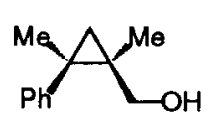

$85 \%(16 \% \theta \theta)$

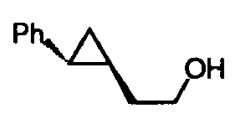

$88 \%(5 \% \theta \theta)$

Finally, an optimized protocol was developed by a survey of $\mathrm{ZnI}_{2}$ sources and stoichiometry. It was found that the in-situ generation of $\mathrm{ZnI}_{2}(100 \mathrm{~mol} \%)$ from diethylzinc and iodine gave the best results. In addition, when the diethylzinc was freshly distilled the enantioselectivities were uniformly higher. A selection of five substrates was chosen to illustrate the generality and superiority of this final protocol (Chart 3). The results of the standard protocol are shown in comparison to the optimized protocol (italic). It is noteworthy that the $\alpha, \beta$-disubstituted substrate did not respond well. 


\section{Chart 3}<smiles>OCC1CC1c1ccccc1</smiles>

$91 \%(80 \% \theta \theta)$ $92 \%(89 \% \theta \theta)$

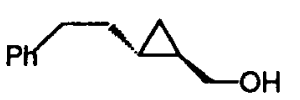

$89 \%(81 \% \theta \theta)$ $88 \%(89 \% \theta \theta)$

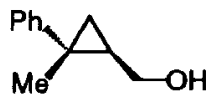

$91 \%(73 \% \theta \theta)$ $92 \%(89 \% \theta \theta)$

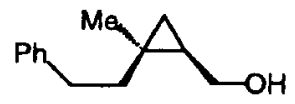

$98 \%(66 \% \theta \theta)$ $89 \%(82 \%$ өe)

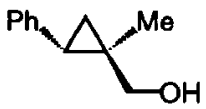

$90 \%(5 \% \theta \theta)$ $91 \%(3 \% \theta \theta)$

A general method for the catalytic asymmetric cyclopropanation of allylic alcohols has been developed. Current efforts are focused on the elucidation of the nature of the reactive zinc-containing species and the development of more selective catalysts.

Acknowledgment. We are grateful to the Upjohn Company for financial support. BLC thanks the Wenner-Gren Center Foundation for a postdoctoral fellowship and SPO thanks the University of Illinois for a Graduate Fellowship.

\section{References}

(1) Doyle, M. P. In Catalytic Asymmetric Synthesis; Ojima, I., Ed.; VCH: New York, 1993; pp 63-99.

(2) Mash, E. A.; Torok, D. S. J. Org. Chem. 1989, 54, 250 and references cited therein.

(3) Mori, A.; Arai, I.; Yamamoto, H.; Nakai, H.; Arai, Y. Tetrahedron 1986, 42, 6447 and referenced cited therein.

(4) Sugimura, T.; Futagawa, T.; Tai, A. Tetrahedron 1990, 46, 5955.

(5) Charette, A. B.; Côté, B.; Marcoux, J.-F. J. Am. Chem. Soc. 1991, 113, 8166. (b) Charette,

A. B.; Juteau, H. J. Am. Chem Soc. 1994, 116, 2651. (c) See also Ukaji, Y.; Nishimura, M.;

Fujisawa, T. Chem. Lett. 1992, 61.

(6) Denmark, S. E.; Edwards, J. P.; Wilson, S. R. J. Am. Chem. Soc. 1992, 114, 2592.

(7) Takahashi, H.; Yoshioka, M.; Ohno, M.; Kobayashi, S. Tetrahedron Lett. 1992, 33, 2575.

(8) (a) Denmark, S. E.; Christenson, B. L.; Coe, D. M.; O'Connor, S. P. Tetrahedron Lett. 1995,

36, 2215. Denmark, S. E.; Christenson, B. L.; O'Connor, S. P. Tetrahedron Lett. 1995, 36, 2219.

(9) Nishimura, J.; Kawabata, N.; Furukawa, J. Tetrahedron 1969, 25, 2647.

(10) For example in the asymmetric, copper-catalyzed cyclopropanation of olefins with diazoacetates, the configuration at the ester group is preserved in both cis and trans isomers (see ref. 1). 